\title{
Influence of Consumer Purchasing Behaviour on the Production Planning of Perishable Food
}

\author{
P. Amorim ${ }^{\mathrm{a}, *}$, A. M. Costa ${ }^{\mathrm{b}}$, B. Almada-Lobo ${ }^{\mathrm{a}}$ \\ ${ }^{a}$ INESC TEC, Faculdade de Engenharia, Universidade do Porto, Rua Dr. Roberto Frias, \\ s/n, 4600-001 Porto, Portugal \\ ${ }^{b}$ Instituto de Ciências Matemáticas e de Computação, Universidade de São Paulo, Av. \\ do Trabalhador São-Carlense, 400, CP. 668, São Carlos, SP 13560-970, Brazil
}

\begin{abstract}
This paper addresses the impact of consumer purchasing behaviour on the production planning of perishable food products for companies operating in the fast moving consumer goods using direct store delivery. The research presented here builds on previous marketing studies related to the effects of expiry dates in order to derive mathematical formulae, which express the age dependent demand for different categories of perishable products. These demand expressions take into account both customer willingness to pay and product quality risk. The paper presents deterministic and stochastic production planning models, which incorporate the customer's eagerness to pick up the fresher products available. Results indicate that model approximations neglecting the fact that customers pick up the fresher products or considering that all products have the same product quality risk have a reduced impact on profit losses. On the other hand, not considering the decreasing customer
\end{abstract}

\footnotetext{
${ }^{*}$ Corresponding author

Email addresses: amorim.pedro@fe.up.pt (P. Amorim), alysson@icmc.usp.br (A. M. Costa), almada.lobo@fe.up.pt (B. Almada-Lobo)
} 
willingness to pay has an important impact both on the profit losses and on the amount of spoiled products.

Keywords: Food Perishability, Production Planning, Consumer

Purchasing Behaviour, Demand Uncertainty

\section{Introduction}

Since the beginning of the 1980s, retailers have gained power over suppliers. One of the most important measures derived from this trend is the direct store delivery (Otto et al., 2009). In this business practice, producers are responsible for all supply chain processes downstream of production until the final customer is reached. Therefore, producers are responsible for selling the products directly at the point of sales, assuring the distribution of the goods bypassing the retailers' warehouses and controlling all merchandising activity. The reported advantages for retailers are related to sales increase due to more intense merchandising activity and out-of-stock reduction. Furthermore, bypassing the retailers' warehouses saves hours of administrative and physical handling of supplier goods. For producers, the benefits come less from a reduction in direct out-of-pocket costs than from being more able to contact directly with their final customers. This enables companies to adjust promotional activities faster and observe and react promptly to market desires for new products.

According to a joint study conducted at the University of Regensburg and SAP on direct store delivery, named Global Direct Store Delivery Analysis $(\mathrm{GDA})^{1}, 24$ out of the 30 largest worldwide fast moving consumer goods com-

\footnotetext{
${ }^{1}$ http://www.sdn.sap.com/irj/scn/go/portal/prtroot/docs/library/uuid/d09e75f9-
} 
panies and 8 out of the 10 largest worldwide food companies have adopted direct store deliveries. In fact, the advantages for the aforementioned producers concerning the adoption of direct store delivery are leveraged when the products handled are perishable (Amorim et al., 2012). This happens to be the case in many food industries. The distribution configuration flowing from direct store delivery is usual a 2 or 3-Tier network linking the production facility to the retailer. Therefore, either products flow directly from facilities to retail stores or there is an intermediate storage decoupling these two stages. If products are highly perishable, a 2-Tier configuration is preferred in order to decrease the amount of freshness lost during distribution. Thus, production models that account for perishability using a more supply chain oriented approach and focus on the consumer purchasing behaviour are expected to provide better overall results when companies use this type of lean practice.

The discussion on the impact of the perishability phenomenon has been gaining importance, especially within the Marketing and Operations Research communities. Marketing and consumer behaviour related studies have focused on understanding the effects that product freshness have on consumption (Sen \& Block, 2009), investigating how customers react to stockouts of highly perishable products (Van Woensel et al., 2007) and the effect of expiration dates on the purchasing behaviour (Tsiros \& Heilman, 2005). In the Operations Research community, the recent reviews of Karaesmen et al. (2011), Akkerman et al. (2010) and Amorim et al. (2011b) covering 
the research conducted in different supply chain planning problems refer to the importance of incorporating perishability explicitly in formulating these problems.

This study considers the perishability definition proposed in Amorim et al. (2011b): "A good, which can be a raw material, an intermediate product or a final one, is called 'perishable' if during the considered planning period at least one of the following conditions takes place: (1) its physical status worsens noticeably (e.g. by spoilage, decay or depletion), and/or (2) its value decreases in the perception of a(n internal or external) customer, and/or (3) there is a danger of a future reduced functionality in some authority's opinion.".

The focus of this paper is on the fast moving food consumer goods subject to physical spoilage. Examples of these products are found in the catering, dairy and processed food industries. The production systems related to these industries involve complex setup sequences, which are often decided by specialised planners according to natural constraints. These characteristics, together with a high inventory rotation, force the collapse of traditional tactical and operational decision planning levels (Bilgen \& Günther, 2009). Within this scope, this paper considers that the producer (that ultimately sells perishable food products to final customers) has no control over the pricing of the products, which is assumed to be fixed within the considered planning horizon. For the readers interested in combining lot sizing and pricing decisions, there are numerous studies on this topic, such as those by Abad (2001, 2003); Chen \& Chen (2005); Chang et al. (2006). In this setting, this study proposes mathematical models that are able to differentiate between differ- 
ent functions of the age dependent demand and/or products with or without a stamped best-before-date. The aim is to bridge the gap between consumer purchasing behaviour and production planning of perishable products by addressing the producers' problem arising from an increasing control over the downstream supply chain. This is indeed the case of many food industries that use direct store delivery and companies that produce and sell in the same establishment (such as bakeries, for example). Therefore, the production planning formulations dealing with perishable products are extended by incorporating consumer purchasing behaviour. This is done by adjusting the product demand, considering inventory depletion and mimicking the consumer's attitude towards perishability. This study considers that demand is influenced by two distinct factors: the decreasing consumer willingness to pay for products with an increasing age and the different demand shapes that are related to the product quality risk. Moreover, it is acknowledged that customers who have the opportunity to choose between equivalent products with different ages will pick the fresher ones.

In the remainder of this article, the papers dealing with production planning of perishable goods are reviewed and the way perishability has been incorporated is analysed. Section 3 describes analytically key issues discovered in the consumer purchasing behaviour theory, such as customer willingness to pay and perceived product quality risk when dealing with perishable food goods. Section 4 presents a deterministic mathematical model and its stochastic extension that considers demand uncertainty. A computational study is conducted in Section 5 to evaluate the impact of incorporating consumer behaviour on the production planning of perishable goods. Finally, the main 
conclusions and future work are discussed in Section 6 .

\section{Production Planning dealing with Perishability Issues}

Only a few papers have focused on production planning problems for perishable goods. However, they are very recent and have been growing in number over the last years, confirming the increasing interest that this topic has been receiving. This section reviews literature closely related to the problem description addressed here. The readers interested in more general tactical / operational production planning research are referred to the reviews of Drexl \& Kimms (1997); Karimi et al. (2003); Zhu \& Wilhelm (2006); Jans \& Degraeve (2008); Buschkühl et al. (2008).

Marinelli et al. (2007) formulate a situation arising in a packaging company producing yoghurt as a hybrid continuous setup lot sizing problem with shared buffers. The authors decompose a relaxed version of the overall problem into a lot sizing problem on tanks (buffers, which store the product recipe) and a scheduling problem on production lines. This decomposition is only possible because the authors neglect setup times (for both tanks and lines) and setup costs (for the lines). They account for perishability by imposing a make-to-order strategy, which is rather hard to follow in the fast moving consumer goods industries. In the case of yoghurt production, Lütke Entrup et al. (2005) develop three models that incorporate shelf-life issues into production planning and scheduling of the packaging stage. Their study uses the block planning concept that delivers a practical means for solving such planning problems (Günther et al., 2006). For the objective

function, they consider a decreasing value for the perishable goods through- 
out the course of their shelf-life. However, the authors acknowledge that the proposed approach is hard to implement in practice since retailers are not willing to pay producers a price based on the remaining shelf-life of the products delivered. Pahl \& Voß (2010) and Pahl et al. (2011) extend well-known discrete lot sizing and scheduling models, such as the general lot sizing and scheduling problem (Fleischmann \& Meyr, 1997) by including deterioration and perishability constraints. They confirm the importance of including such constraints, which may reduce the solution search space. In Amorim et al. (2011a), a multi-objective framework is used with one objective related to production costs and another related to freshness. This problem is solved by using a hybrid multi-objective evolutionary algorithm and the result of the lot sizing and scheduling problem is a Pareto front trading off these two key performance indicators. The conclusions indicate that only by considering perishability in one objective function, it is possible to achieve a much higher freshness standard at the expense of an additional small cost.

In all aforementioned papers, demand is seen as an external, dynamic and deterministic parameter that does not have a real connection to the perishable nature of the product. This link is evidenced in our study by bringing in the research on consumer purchasing behaviour related to food products into production planning models. Moreover, the influence of demand uncertainty on such setting is also investigated. Therefore, further insights are expected on the impact of perishable food goods on production planning decisions. 


\section{Modelling Consumer Purchasing Behaviour}

This section derives the demand expressions that are function of the product age, which will feed the production planning formulations. The basis of the modelling presented here is the consumer purchasing behaviour for perishable products described in Tsiros \& Heilman (2005). To the best of our knowledge, this is the only study on the consumer purchasing behaviour that investigates the effects of perishability on the purchasing pattern of customers across different perishable products. In this study, it was possible to conclude that customer willingness to pay (WTP) decreases throughout the course of the products' shelf-life. Moreover, this decrease follows a linear function for products with a low product quality risk (PQR), whereas the WTP follows an exponential negative function for products with a high PQR. In the sam-

ple, products such as lettuce, milk, carrots and yoghurt were considered low PQR products, while beef and chicken were described as high PQR products. Note that PQR is defined as the expected negative utility associated with the product as it reaches its expiry date, and WTP is the maximum price a customer is willing to pay for a given product at a given point in time. It is important to highlight that the customer WTP for a perishable product does not have to be strictly related to the organoleptic condition of its content. The study by Tsiros \& Heilman (2005) does not make any assumption regarding the perishable nature of the products. Therefore, its conclusions should be applicable to products with fixed or random lifetime (Nahmias, 1982).

In order to understand how demand varies with an increasing age of the product for a fixed list price, two relations need to be explicitly understood: 
firstly, how the WTP (price) varies with an increasing age and, secondly, what is the relation between demand and price. Let us denote the function describing the behaviour of price $p$ for each age $a$ as $p=f(a)$ and assume that the first order derivative of this function is independent of the demanded quantity. Furthermore, it is necessary to consider a demand function for a product of age 0 (fresher state) $d^{0}=h^{0}(p)$, where $d$ denotes demand, which also has the same first order derivative across the functions for each different age. Hence, given that $f$ is a monotonic decreasing function, the respective $h^{a}$ function for a product of age $a$ is obtained by shifting function $h^{0}$ by $f(0)-f(a)$. After this, it is possible to define the demand in terms of price and age as:

$$
d(p, a)=h^{0}(p+f(0)-f(a))
$$

Figure 1 provides a graphical representation of the aforementioned functions.

Within the price/demand range considered in the medium-term production planning and following the same reasoning of the vast majority of the economic studies aiming at finding the price elasticity of demand for different products, a constant value for elasticity was used that will only depend on the product being considered. Thus the constant elasticity demand function $h^{0}(p)$ reads:

$$
h^{0}(p)=C p^{\epsilon}
$$

where $C$ stands for a constant and $\epsilon$ for the elasticity. Therefore, 


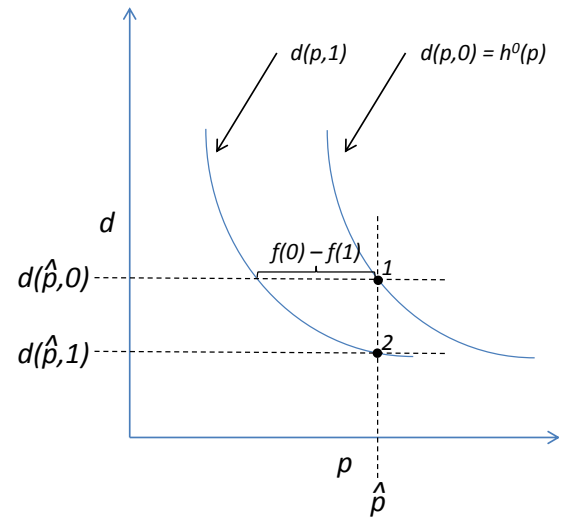

Figure 1: Graphical interpretation of the mathematical relation between function $f, d$ and $h^{0}$. Point 1 gives the demand for a list price $\hat{p}$ of a product with 0 units of age and point 2 for a product with 1 unit of age.

$$
d(p, a)=C(p+f(0)-f(a))^{\epsilon}
$$

Assuming that the demand is known for each product in its fresher state $\left(d^{0}\right)$ for a given list price $(\hat{p})$, which corresponds to the usual setting in production planning problems, constant $C$ can be written as:

$$
C=\frac{d^{0}}{\hat{p}^{\epsilon}} .
$$

Replacing this expression in equation (3) gives:

$$
d(p, a)=d^{0}\left(\frac{p+f(0)-f(a)}{\hat{p}}\right)^{\epsilon} .
$$

To fully describe the demanded quantity as a function of the age of the product for a given list price, one only needs to describe the $f$ functions, which are empirically studied in Tsiros \& Heilman (2005). Based on the observations of the WTP for different products at different ages in this study, 
it is possible to derive mathematical functions describing such behaviour. In order to analyse a wider range of products than those considered in that study, a new function establishing a concave WTP profile is proposed that is used for products with a low PQR, such as bread. The linear and convex demand profiles proposed by Tsiros \& Heilman (2005) are used for products with medium and high PQR, respectively. All these functions exhibit a similar behaviour as they are monotonically decreasing, having its maximum value for the product with a maximum freshness $\left(p^{0}\right)$ and a value of 0 at the end of shelf-life $u$. The closeness of the WTP to 0 monetary units as the product reaches its shelf-life is controlled by parameter $\alpha$, which is independent of the function shape (linear, concave or convex). Therefore, this parameter, which varies between 0 and 1, will represent the customer sensitivity to the decaying freshness of the product. If $\alpha=0$, the customer attributes a constant value to the perishable product and if $\alpha>0$, the customer gives an increasing importance to product freshness until the point when $\alpha=1$, which corresponds to a customer that towards the end of the shelf-life will be willing to pay 0 monetary units for the product.

Equations (5)-(7) and Figure 2 represent the linear, concave and convex WTP shapes.

$$
\begin{gathered}
\text { Linear } f_{\text {linear }}(a)=p^{0}-\frac{\alpha p^{0} a}{u-1} \\
\text { Concave } f_{\text {concave }}(a)=p^{0}-\frac{\alpha p^{0} a}{u-1}\left(\frac{a}{u-1}\right) \\
\text { Convex } f_{\text {convex }}(a)=p^{0}-\frac{\alpha p^{0} a}{u-1}\left(2-\frac{a}{u-1}\right)
\end{gathered}
$$




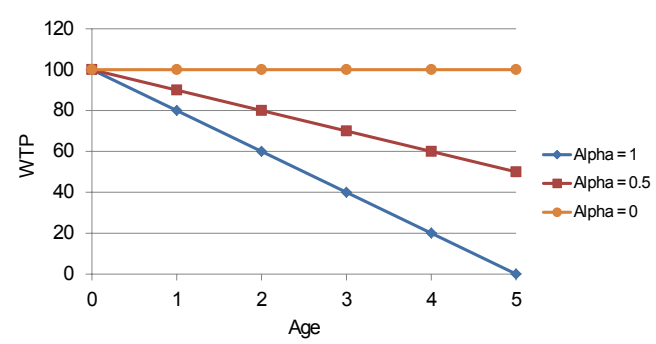

(a) linear

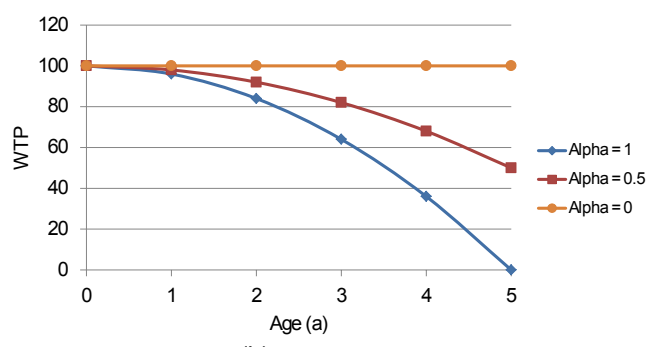

(b) concave

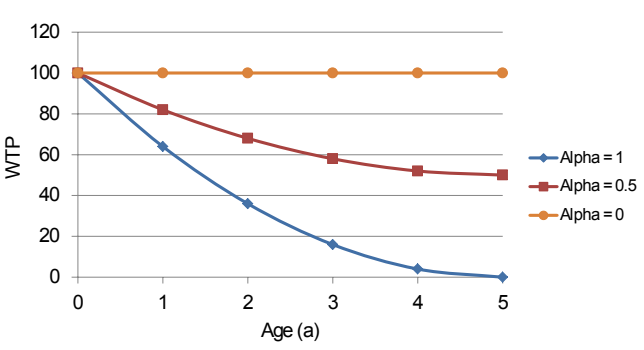

(c) convex

Figure 2: Impact of different $\alpha$ when customers WTP function is (a)linear, (b)concave, (c)convex.

In the figures, all functions consider a WTP for the product at its fresher state $p^{0}$ to be equal to 100 and shelf-life $u$ equal to 6 . Note that in each function the price is represented until age 5 , since at age 6 the products spoil and can no longer be sold. A concave demand function means that the customers become more sensitive towards the end of the shelf-life and their WTP for each age of the product is always above the linear function. On the other hand, when the WTP function is convex, the WTP drops very fast as soon as the product is produced. In this case the curve is always below the linear function. This is actually the case in processed fish, since customers are aware of the increased risk of consuming it in a less fresh state. The linear shape represents products to which consumers attribute a medium risk, such as yoghurt. 


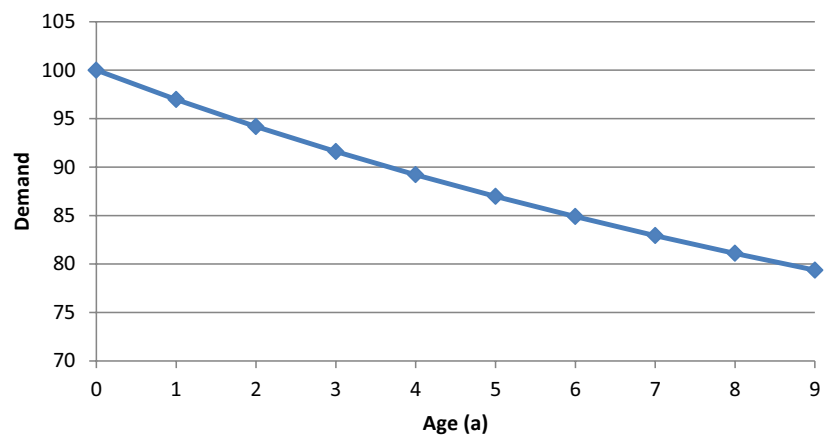

Figure 3: Example of the demand curve for lettuce over its shelf-life of 10 days, starting at 100 units. According to Tsiros \& Heilman (2005) lettuce has a medium PQR, $\alpha=0.62$, $\hat{p}=2.49$ and $p^{0}=2.86$; and according to Andreyeva et al. (2010) it has $\epsilon=-0.58$.

Finally, the demand functions for different ages can be described by replacing $f(a)$ functions in $d(p, a)$ functions. Equations (8)-(10) represent the linear, concave and convex demand shapes for list price $\hat{p}$.

$$
\begin{gathered}
\text { Linear } d_{\text {linear }}(\hat{p}, a)=d^{0}\left(\frac{\hat{p}+\frac{\alpha p^{0} a}{u-1}}{\hat{p}}\right)^{\epsilon} \\
\text { Concave } d_{\text {concave }}(\hat{p}, a)=d^{0}\left(\frac{\hat{p}+\frac{\alpha p^{0} a}{u-1}\left(\frac{a}{u-1}\right)}{\hat{p}}\right)^{\epsilon} \\
\text { Convex } d_{\text {convex }}(\hat{p}, a)=d^{0}\left(\frac{\hat{p}+\frac{\alpha p^{0} a}{u-1}\left(2-\frac{a}{u-1}\right)}{\hat{p}}\right)^{\epsilon}
\end{gathered}
$$

In order to illustrate the behaviour of the demand curves the examples of lettuce and beef products are provided in Figures 3 and 4, respectively.

\section{Deterministic and Stochastic Mathematical Models}

In this section, a deterministic production planning model that considers the consumer purchasing behaviour is formulated to deal with perishable food 


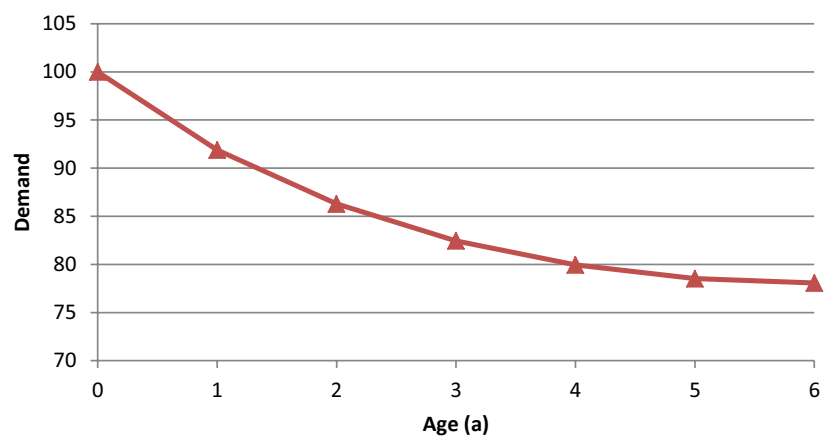

Figure 4: Example of the demand curve for beef over its shelf-life of 7 days, starting at 100 units. According to Tsiros \& Heilman (2005) beef has a high PQR, $\alpha=0.52, \hat{p}=2.68$ and $p^{0}=2.52$; and according to Andreyeva et al. (2010) it has $\epsilon=-0.75$.

goods. This formulation is then extended to incorporate demand uncertainty, and a two-stage stochastic programming with recourse model is proposed.

Consider products $k=1, \ldots, K$ that are produced based on a certain recipe forming a block $j=1, \ldots, N$. There is only one recipe to produce each product and, therefore, a product is assigned to one block only. Hence, for each block $j$ there is a set $\left[K_{j}\right]$ of products $k$ related to it. Blocks are to be scheduled on parallel production lines $l=1, \ldots, L$ over a finite planning horizon consisting of periods $t=1, \ldots, T$ with a given length. This length is related to the company's practice of measuring external elements, such as demand (thus, periods correspond to days, weeks or months in most cases). The production sequence is defined a priori obeying to natural sequences with product families in mind. These tight production conditions are frequent in the fast moving consumer goods industries and reflect the technological and batch requirements that these industries face. Hence, this study relies on the block planning approach that predefines the sequence of the blocks and products beforehand, minimising the setup times and costs according 
to the planner expertise (Günther et al., 2006). Consider, for example, the production of beverages. If one is to switch over to a similar package where the label is the only changing element, then a minor setup is to be performed. However, if the beverage to be produced also changes, then a major setup is required.

Consider the following indices, parameters and decision variables that are used both in the deterministic and stochastic formulations.

\section{Indices}

$l \in[L] \quad$ parallel production lines

$i, j \in[N]$ blocks

$k \in\left[K_{j}\right] \quad$ products

$t \in[T] \quad$ periods

$a \in[A] \quad$ ages (in periods) 


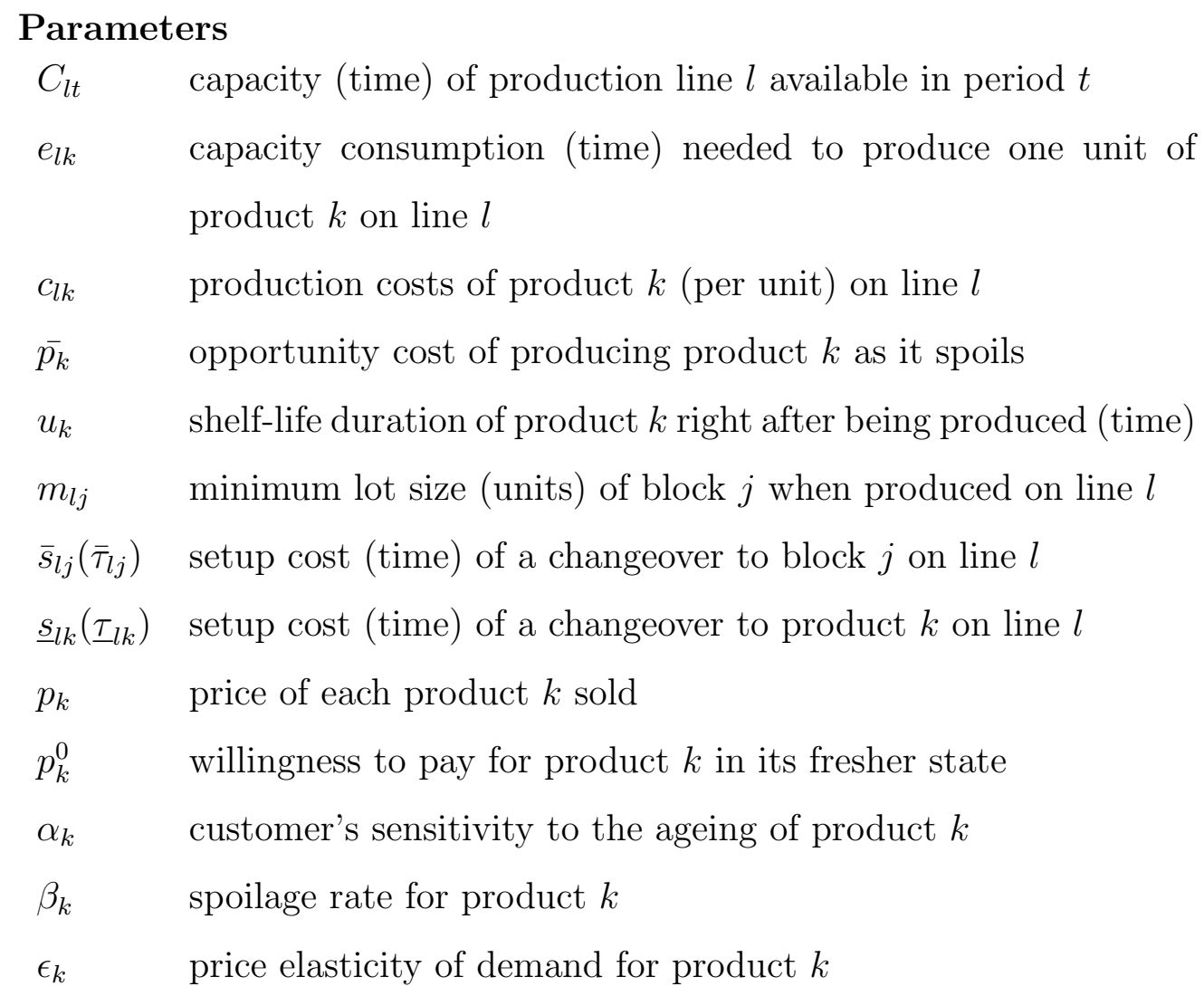

\section{Production Related Decision Variables}

$q_{l k t}$ quantity of product $k$ produced in period $t$ on line $l$

$p_{l k t}$ equals 1 , if line $l$ is set up for product $k$ in period $t$ ( 0 otherwise)

$y_{l j t} \quad$ equals 1 , if line $l$ is set up for block $j$ in period $t$ ( 0 otherwise)

Figure 5 exemplifies the relation between blocks and products in the production planning approach used.

\subsection{Deterministic Demand}

To formulate the production planning model for perishable products with a deterministic demand (PP-P-D), it is necessary to define the decision vari- 


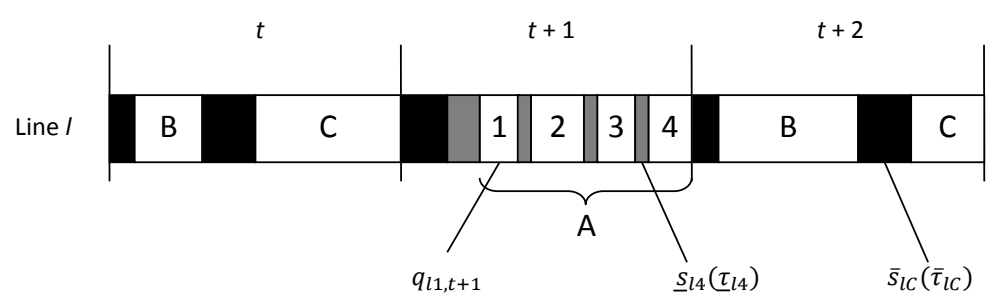

A, B,... - Blocks
$1,2, \ldots$ - Products

Figure 5: Relation between blocks, products, major and minor setups.

ables related to the demand fulfilment. Firstly, the parameter $d_{k t}^{a}$ (demand for product $k$ with age $a$ in period $t$ ) is calculated in a preprocessing step based on one of the described functions (8) - (10) (cf. Section 3) depending on the specific PQR and the parameters defined. The decision variables that shall be added to those already defined:

$w_{k t}^{a}$ initial inventory of product $k$ with age $a$ available at period $t, a=$ $0, \ldots, \min \left\{u_{k}, t-1\right\}$

$\psi_{k t}^{a} \quad$ fraction of the demand for product $k$ delivered with age $a$ at period $t, a=0, \ldots, \min \left\{u_{k}-1, t-1\right\}$

$\theta_{k t}^{a} \quad$ equals 1 , if inventory of product $k$ with age $a$ is used to satisfy demand in period $t$ ( 0 otherwise), $a=1, \ldots, \min \left\{u_{k}-1, t-1\right\}$

Note that these three sets of decision variables are only instantiated for certain domains to ensure that no perished products are kept in stock or used to fulfil demand. Further note that $\left[A_{w}\right]=\left\{a \in \mathbb{Z}_{0}^{+} \mid a \leq \min \left\{u_{k}, t-1\right\}\right\}$ and $\left[A_{\psi, \theta}\right]=\left\{a \in \mathbb{Z}_{0}^{+} \mid a \leq \min \left\{u_{k}-1, t-1\right\}\right\}$, the utility of these sets will be clearer when developing the constraints. 
Figure 6 represents the relation between the demand fulfilment decision variables for an example with product $k$ having production only in day $t$. The demand is completely fulfilled in this period and partially met in period $t+1$ with a stock of age 1 . The demand fulfilment decision variables are related to the simple plant location (SPL) reformulation (Krarup \& Bilde, 1977), which has been used, for example, by Lütke Entrup et al. (2005) in the context of yoghurt production. There are other works that use a similar approach based on the evolving quality of stocks, which does not have to be strictly correspondent to the age of the products (Rong et al., 2011; Ahumada \& Villalobos, 2011). The way we use explicitly age as a driver to the perceived quality and the inventory balance constraints are more in line to the work of Costa et al. (2013), which deals with the sustainable vegetable crop supply problem with perishable stocks.

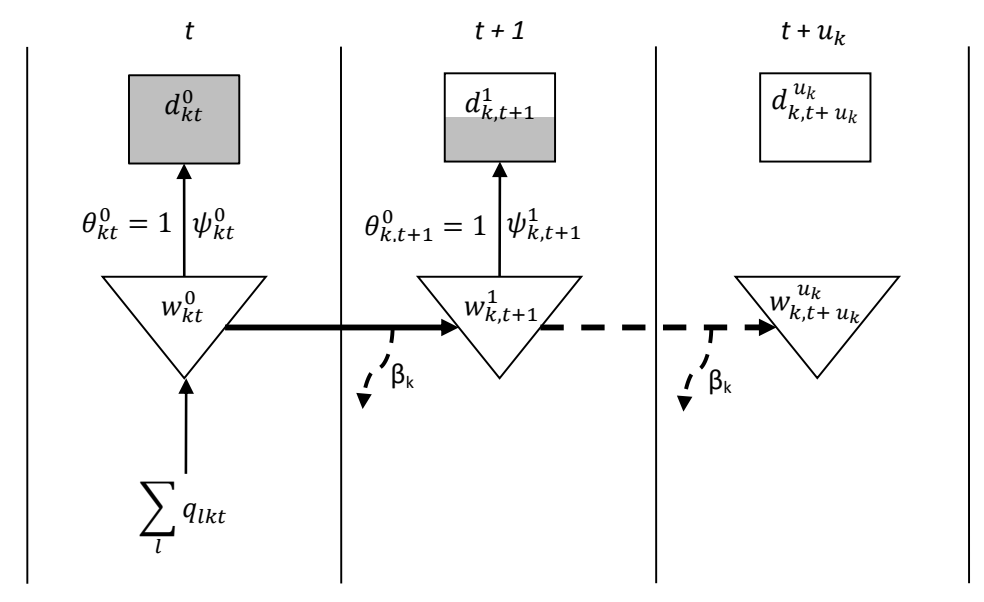

Figure 6: Relation between demand fulfilment variables.

The deterministic model reads: 


\section{PP-P-D}

$$
\begin{array}{r}
\max \sum_{k, t, a} p_{k} \psi_{k t}^{a} d_{k t}^{0}-\sum_{l, j, t} \bar{s}_{l j} y_{l j t}-\sum_{l, k, t}\left(\underline{s}_{l k} p_{l k t}+c_{l k} q_{l k t}\right) \\
-\sum_{k, t, a} \bar{p}_{k} \beta_{k}\left(w_{k t}^{a}-\psi_{k t}^{a} d_{k t}^{0}\right)
\end{array}
$$

subject to:

$$
\begin{gathered}
\sum_{a \in\left[A_{\psi, \theta}\right]} \psi_{k t}^{a} \leq 1 \quad \forall k \in[K], t \in[T] \\
\psi_{k t}^{a} d_{k t}^{0} \leq d_{k t}^{a} \quad \forall k \in[K], t \in[T], a \in\left[A_{\psi, \theta}\right] \\
\psi_{k t}^{a} \leq \theta_{k t}^{a} \quad \forall k \in[K], t \in[T], a \in\left[A_{\psi, \theta}\right] \\
w_{k t}^{a-1}-\psi_{k t}^{a-1} d_{k t}^{0} \leq\left(1-\theta_{k t}^{a}\right) M \quad \forall k \in[K], t \in[T], a \in\left[A_{\psi, \theta}\right] \backslash\{0\} \\
w_{k t}^{a}=\left(w_{k, t-1}^{a-1}-\psi_{k, t-1}^{a-1} d_{k, t-1}^{0}\right)\left(1-\beta_{k}\right) \quad \forall k \in[K], t \in[T+1], a \in\left[A_{w}\right] \backslash\{0\} \\
\sum_{l} q_{l k t}=w_{k t}^{0} \quad \forall k \in[K], t \in[T] \\
p_{l k t} \leq y_{l j t} \quad \forall l \in[L], j \in[N], k \in\left[K_{j}\right], t \in[T] \\
e_{l k} p_{l k t} \quad \forall l \in[L], k \in[K], t \in[T]
\end{gathered}
$$




$$
\begin{gathered}
\sum_{j} \bar{\tau}_{l j} y_{l j t}+\sum_{k}\left(\underline{\tau}_{l k} p_{l k t}+e_{l k} q_{l k t}\right) \leq C_{l t} \quad \forall l \in[L], t \in[T] \\
\sum_{k \in\left[K_{j}\right]} q_{l k t} \geq m_{l j} y_{l j t} \quad \forall l \in[L], j \in[N], t \in[T] \\
\psi_{k t}^{a}, w_{k t}^{a}, q_{l k t} \geq 0 ; p_{l k t}, y_{l j t}, \theta_{k t}^{a} \in\{0,1\}
\end{gathered}
$$

Objective function (11) maximises the profit of the producer over the planning horizon. Major setup costs between blocks, minor setup costs between products and variable production costs are subtracted from the sum of the revenue of each unit sold. Moreover, the cost of spoiled products is also subtracted and valued through the opportunity cost $\bar{p}_{k}$. This opportunity cost corresponds to the revenue yielded by the best alternative that could have been produced and sold instead of producing product $k$ that got spoiled. However, it may also be regarded, in a more quantifiable manner, as a disposal cost for each unit of perished inventory that has to be properly discarded.

Equations (12) prevent the sum of all sold products of different ages from exceeding the total demand for the product in the fresher state. Then, equations (13) do not permit the quantity of sold products of a given age to be above the demand curve derived from the customer WTP. However, with only these two constraints, the seller is able to assign the optimal inventory quantities with different ages to customers in order to maximise profit. With constraints (14) and (15) this situation is avoided by mimicking the more instinctive consumer purchasing behaviour related to perishable food products that will drive customers to pick products with the highest degree 
of freshness (Broekmeulen \& van Donselaar, 2009). Thus, constraints (14) turn the value of $\theta_{k t}^{a}$ into 1 whenever the inventory of a given product $k$ in period $t$ with age $a$ is used to satisfy the demand. The value of variable $\theta_{k t}^{a}$ is used in equations (15) to ensure that an older inventory can only be used after depleting the fresher inventory. Note that parameter $M$ denotes a large number. Constraints (16) establish the inventory balance constraints, which are modelled here in a propagation form, updating the age of the inventories throughout the planning horizon. The products in inventory may have either a fixed lifetime expressed by a stamped best-before-date (milk and meet) or a random lifetime if no stamp is found (fruits and vegetables). The quantity throughout the time of these latter products is commonly modelled as subject to exponential decay. In constraints (16) this spoilage phenomenon is controlled by parameter $\beta_{k}$. On the one hand, if $\beta_{k}=0$, the product in inventory will spoil only at the end of its shelf-life. On the other hand, if $\beta_{k}>0$, then inventory is subject to exponential decay. The higher the $\beta_{k}$ value, the higher the amount of spoilage from one period to the next.

Equations (17) establish that the production of a product for a certain period on all is equal to the available stock of that same product with age 0. These constraints link the production flow and the demand fulfilment requirements. Constraints (18) and (19) ensure that in order to produce a certain product, the necessary setups are performed for the corresponding recipe and product, respectively. Moreover, each block is subject to a minimum lot size (21). Finally, constraints (20) limit the use of the capacity with setups and production in each period. 


\subsection{Uncertain Demand}

In this section, the previous deterministic model is extended by incorporating demand uncertainty, which is typical in food products. These products are subject to a very intense promotion activity with uncertain outcomes and companies in this business are very keen to launch new products to the market (Lütke Entrup, 2005). The main motivation to study the stochastic version of the deterministic model with an uncertain demand is the fact that in the deterministic setting, unless minimum lot sizes are of a very significant size when compared to the demand orders, there will never be any spoiled product, for the case of products with a fixed lifetime $\left(\beta_{k}=0\right)$. In fact, the production quantities are such that after fulfilling the predicted demand no inventory lasts until the end of its shelf-life. However, in real-world problems, consumer goods industries of food products face enormous challenges to reduce the amount of spoilage derived from not selling products.

A two-stage stochastic programming with recourse model is proposed here in order to cope with demand uncertainty. Stochastic programming models with recourse are adequate when decisions may be decoupled into two separate stages: first-stage decisions are made before the uncertainty is unveiled, and second-stage decisions take place after uncertainty has occurred. Thus, second-stage decisions may be able to correct some imprecisions coming from the myopic decisions made in the first-stage. For an introductory study on stochastic programming the readers are referred to Birge \& Louveaux (2011). Justification for treating uncertainty with this methodology is given, for example, in Sodhi (2005). This work deals with the supply chain planning problem in a real-world electronics company that has an uncertain demand. 
In this problem, the first-stage decisions are related to the production planning and scheduling of blocks and products $(q, p, y)$, and the second-stage decisions are responsible for managing the ongoing perishable inventory and demand fulfilment $(w, \psi, \theta)$. The motivation for such division comes from the fact that most producers decide on the production plans before actually knowing the accurate demand. For example, in the yoghurt production, in which the final packing stage is connected to the bulk recipe stage that has significant lead times, the production planning and sequencing are performed weeks in advance (first-stage). Afterwards, the inventory at the retailers or distribution centers is used to hedge against demand variability (secondstage).

The stochastic demand is modelled using a set $[V]$ of discrete scenarios $v=1, \ldots, V$, each of which with an associated probability $\phi^{v}$, such that

$\phi^{v}>0, \forall v$ and $\sum_{v} \phi^{v}=1$. In order to formulate the stochastic model, it is necessary to extend the domains of the demand parameter and of every demand fulfilment variable defined at the beginning of Section 4.1 by adding a new index $v$ related to the probable scenarios $\left(w_{k t}^{a v}, \psi_{k t}^{a v}, \theta_{k t}^{a v}\right)$.

The two-stage stochastic programming with recourse model tackling the production planning problem for perishable products with uncertain demand (PP-P-U) may be formulated as follows:

\section{PP-P-U}

$$
\max \sum_{v} \phi^{v}\left[\sum_{k, t, a}\left(p_{k} \psi_{k t}^{a v} d_{k t}^{0 v}-\overline{p_{k}} \beta_{k}\left(w_{k t}^{a v}-\psi_{k t}^{a v} d_{k t}^{0 v}\right)\right)-\sum_{k, t} \overline{p_{k}} w_{k t}^{u_{k}, v}\right]
$$




$$
-\sum_{l, j, t} \bar{s}_{l j} y_{l j t}-\sum_{l, k, t}\left(\underline{s}_{l k} p_{l k t}+c_{l k} q_{l k t}\right)
$$

subject to:

$$
\begin{gathered}
\sum_{a \in\left[A_{\psi, \theta}\right]} \psi_{k t}^{a v} \leq 1 \quad \forall k \in[K], t \in[T], v \in[V] \\
\psi_{k t}^{a v} d_{k t}^{0 v} \leq d_{k t}^{a v} \quad \forall k \in[K], t \in[T], a \in\left[A_{\psi, \theta}\right], v \in[V] \\
\psi_{k t}^{a v} \leq \theta_{k t}^{a v} \quad \forall k \in[K], t \in[T], a \in\left[A_{\psi, \theta}\right], v \in[V] \\
w_{k t}^{a-1, v}-\psi_{k t}^{a-1, v} d_{k t}^{0 v} \leq\left(1-\theta_{k t}^{a v}\right) M \quad \forall k \in[K], t \in[T], a \in\left[A_{\psi, \theta}\right] \backslash\{0\}, v \in[V] \\
w_{k t}^{a v}=\left(w_{k, t-1}^{a-1, v}-\psi_{k, t-1}^{a-1, v} d_{k, t-1}^{0 v}\right)\left(1-\beta_{k}\right) \quad \forall k \in[K], t \in[T+1], a \in\left[A_{w}\right] \backslash\{0\}, v \in[V] \\
\sum_{l} q_{l k t}=w_{k t}^{0 v} \quad \forall k \in[K], t \in[T], v \in[V] \\
\quad(18)-(21) \\
\psi_{k t}^{a v}, w_{k t}^{a v}, q_{l k t} \geq 0 ; p_{l k t}, y_{l j t}, \theta_{k t}^{a v} \in\{0,1\}
\end{gathered}
$$

In the stochastic problem the objective function differs from the one in the deterministic model (11) not only because it accounts for the different 
demand scenarios, but also because it tries to minimise the spoilage of products that have reached the end of the shelf-life without being sold. These products are obtained in a straightforward manner with the proposed formulation since they correspond to the inventory that reaches an age of $u_{k}$ (given by $\left.w_{k t}^{u_{k}, v}\right)$. It is important to remember that if this spoilage term related to products reaching their shelf-lives had been included in the deterministic objective function (11), its value would have been null (if the minimum lot sizes were small enough in comparison to the demand orders).

Equations (24)-(29) have the same meaning as equations (12)-(17) described in the deterministic model, except that they have been extended to deal with the different demand scenarios. The remaining constraints related to the production planning are exactly the same as the ones already described in the deterministic model (cf. Section 4.1).

\section{Computational Study}

The computational study aims at understanding the impact of the consumer purchasing behaviour on the production planning of perishable food goods from several perspectives. Firstly, the influence of the age dependent

demand, secondly, the influence of the fact that customers are eager to pick up the fresher products and, finally, the effect of faithfully representing product quality risks in the model. Therefore we focus on:

1. Age dependent demand (ADD)

(a) the importance of considering age dependent demand as theorised in the literature on consumer purchasing behaviour; 
(b) the amount of spoiled products caused by neglecting age dependent demand.

2. Customer's eagerness for fresher products (CFP)

(c) the importance of considering customer's eagerness to pick up the fresher products available;

(d) the amount of spoiled products caused by the non-consideration of the customers' characteristic of picking up the fresher products.

3. Product quality risk (PQR)

(e) the impact of neglecting different PQRs, assuming a medium PQR for all products.

The aim is to analyse these three perspectives in different production settings, where the mix of products with different characteristics such as PQR varies. Moreover, it is important to assess the sensitivity of the results to variations in key parameters such as $\alpha_{k}$, which is related to the customer's sensitivity to the ageing process of the product and $u_{k}$ that specifies the quantity of periods that a product is in a sellable state. All generated instances are solved in less than 20 minutes by the mixed-integer programming solver CPLEX 12.1 on a PC with 4 GB of RAM and an Intel Core i5 2.67 $\mathrm{GHz}$ processor running on Windows 7.

\subsection{Data Generation}

The instance generator uses real data for the consumer purchasing behaviour and random data for the production system related parameters.

The real data related to the consumer purchasing behaviour for six different perishable food goods (for instance, lettuce and milk) is based on Tsiros 
Table 1: Data related to consumer purchasing behaviour.

\begin{tabular}{cccccccccc} 
Block & $u_{k}$ & $p_{k}$ & $\bar{p}_{k}$ & $p_{k}^{0}$ & $\alpha_{k}$ & PQR & $\epsilon_{k}$ & $c_{l k}$ & Setting \\
\hline 1 - Lettuce & 10 & 2.49 & 1.245 & 2.86 & 0.62 & Medium & -0.58 & 0.25 & $\mathbf{1}$ \\
2 - Carrots & 21 & 1.69 & 0.845 & 3.12 & 0.78 & Medium & -0.58 & 0.17 & $\mathbf{1}$ \\
3 - Milk & 14 & 2.7 & 1.35 & 4.05 & 0.82 & Medium & -0.59 & 0.27 & $\mathbf{2}$ \\
4- Yogurt & 21 & 0.62 & 0.31 & 1.14 & 0.77 & Medium & -0.65 & 0.06 & $\mathbf{2}$ \\
5 - Chicken & 7 & 2.99 & 1.495 & 2.78 & 0.50 & High & -0.68 & 0.30 & $\mathbf{3}$ \\
6 - Beef & 7 & 2.68 & 1.34 & 2.52 & 0.52 & High & -0.75 & 0.27 & $\mathbf{3}$
\end{tabular}

\& Heilman (2005) and Andreyeva et al. (2010). The data provided in Table 1 allows the generation of three different production settings with two PQR combinations. Settings 1 and 2 (medium PQRs) must meet demand for Lettuce and Carrot products, and for Milk and Yoghurt products, respectively. Setting 3 (high PQRs) meets demand for Chicken and Beef products. For all products, $\bar{p}_{k}$ and $c_{l k}$ are obtained by multiplying price $p_{k}$ by 0.5 and 0.1 , respectively.

Each of these real goods is related to one block and for each of the six blocks there are four products corresponding to different packaging.

A methodology similar to that of Haase \& Kimms (2000) was used to generate the remaining parameters for each setting. Therefore, $L$ was set to 1 and for all products $e_{l k}=1, m_{l j}=1$. This study also considers that all products are packaged and stamped with a best-before-date $\left(\beta_{k}=0\right)$ and that no initial inventory exists. Remark that final inventories may exist due to minimum lot sizes or demand uncertainty. The number of periods $T$ is 20 . For all products in all settings, the fresher state demand $d_{k t}^{0}$ was assumed to be 0 with a probability of 0.25 . In case the demand was not 0 , it was assigned 
a random number using the interval $[40,60]$ of the uniform distribution. The setup costs and times between blocks $\left(\bar{s}_{l j}\right.$ and $\left.\bar{\tau}_{l j}\right)$ were randomly chosen in the interval $[5,10]$. For the setup cost of products $\left(\underline{s}_{l j}\right.$ and $\left.\underline{\tau}_{l j}\right)$ the interval $[1,4]$ is used and all blocks have products with the same product setup values. The capacity per period $C_{l t}$ is determined according to:

$$
C_{l t}=\frac{\sum_{k} d_{k t}^{0}}{U}, \quad \forall l, t
$$

where the capacity utilisation $U$ is 0.6 . It is important to highlight that the utilization of capacity is underestimated, as setup times do not influence the value of $C_{l t}$, and that across all production settings the capacity utilisation is very similar.

To set the stochastic model parameters, 3 scenarios were defined, all with the same probability $\phi^{v}=1 / 3$. In the average scenario the demand for the products at their fresher state $d_{k t}^{0}$ is exactly the same as the values used to feed the deterministic model. The other two scenarios have a demand for the fresher products that corresponds to an increase and a decrease of $30 \%$ in the average values of $d_{k t}^{0}$.

To obtain the complete set of data of the demand for all possible product ages for the deterministic and stochastic models $\left(d_{k t}^{a}\right.$ and $\left.d_{k t}^{a v}\right)$, expressions (8) and (10) were used for products with medium and high PQR, respectively. For example, for the Lettuce block in the deterministic case, the following expression based on equation (8) was used:

$$
d_{\text {Lettuce, } t}^{a}=d_{\text {Lettuce, } t}^{0}\left(\frac{2.49+\frac{0.62 \cdot 2.86 \cdot a}{10-1}}{2.49}\right)^{-0.58}
$$

Afterwards, the complete demand parameter $d_{\text {Lettuce, } t}^{a}$ was obtained by 
plugging in the formula $d_{\text {Lettuce,t }}^{0}$ already (randomly) computed and varying the age $a$,

Regarding the sensitivity analysis, for each of the 3 production Settings, the average value of $\alpha_{k}$ (Alpha) was varied by means of increasing (Alpha+) / decreasing (Alpha-) it $25 \%$ and the average value of $u_{k}(\mathrm{u})$ was varied using the same methodology $(\mathrm{u}+$ and $\mathrm{u}-)$.

\subsection{Results and Discussion}

In order to streamline the flow of this section, the results are organised along the five different analysis ((a) to (e)) presented in the beginning of Section 5. In each perspective the main results are discussed. The methodology to quantify the impact of neglecting the consumer purchasing behaviour in each perspective is the same. The results considering the respective feature (A) are compared to the results in which the feature is neglected (B). To obtain (A), the entire model is solved considering the feature under analysis. To obtain (B), firstly the production related decisions $(q, p, y)$ are optimised without considering the feature and, afterwards, with the production decision variables fixed, the model is solved again with the actual customer behaviour unveiled. In this second step, the demand fulfilment variables $(w, \psi, \theta)$ are released.

\subsubsection{Importance of considering age dependent demand (ADD)}

This perspective analyses the impact on profit caused by neglecting the age dependent demand of perishable food goods for companies using direct store delivery in a deterministic scenario (Section 4.1). Results obtained when considering age depend demand are compared against results obtained 
when the production plan is decided without this acknowledgement. Figures 7 and 8 show the percentage objective function loss due to not considering the age dependent demand.

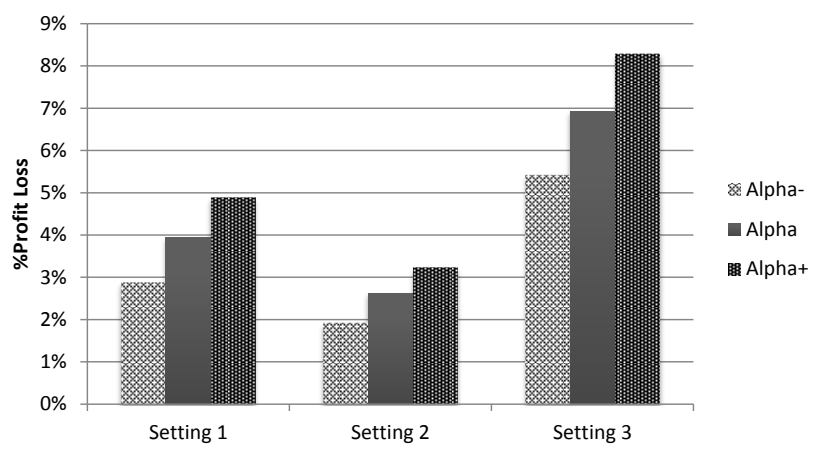

Figure 7: Profit loss due to neglecting age dependent demand for all Settings varying $\alpha_{k}$.

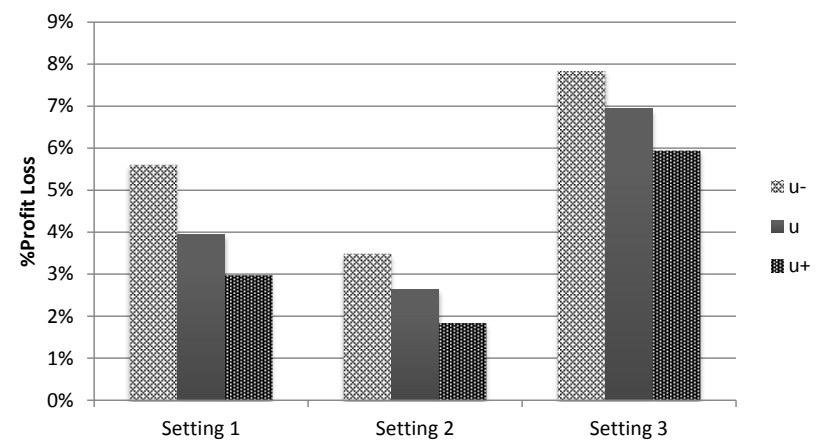

Figure 8: Profit loss due to neglecting age dependent demand for all Settings varying $u_{k}$.

Overall, neglecting the age dependent demand resulted in a significant profit loss. More sensitive customers yielded higher losses, products with lower perceived risk, such as vegetables, have a lower impact on the profit and longer shelf-lives smooth profit losses. Thus, for example, if producers are able to reduce the perceived quality risk, an increase of revenue is expected 
by increasing the total demand throughout the products' shelf-life. It is also important to highlight that there is a considerable interaction between PQR and the customer's exigency. Therefore, as customers become more sensitive to perishability, the impact of product perceived risk tends to increase. For example, if the producer is serving a retailer with very demanding customers, it is important to decrease the product quality risk as much as possible (besides delivering very fresh products). In the case of packaged food goods, such as yoghurt, this can be achieved by filling the product in glass containers instead of plastic ones (Dyllick, 1989). Moreover, packaging can also have a positive effect on profit in case products' shelf-life is increased (see Figure 8).

\subsubsection{Amount of spoiled products caused by not considering age dependent demand $(A D D)$}

As previously stated, a deterministic scenario will hardly yield a solution with spoiled products, unless minimum lot sizes are of a significant size. Therefore, the stochastic model is used to assess this impact. The amount of spoilage obtained when the first-stage variables $(q, p, y)$ are optimised for a constant demand throughout the shelf-life of the products is compared to the amount of spoilage incurred when both first $(q, p, y)$ and second-stage variables $(w, \psi, \theta)$ take into account the age dependent demand. Figures 9 and 10 present the amount of spoilage for all instances when planning with and without age dependent demand. Notice that results for instances of Setting 2 are left out as there was no spoilage incurred, because the shelflives of the products in this Setting are close to the length of the planning horizon.

The results for Settings 1 and 3 show that the amount of spoilage is 


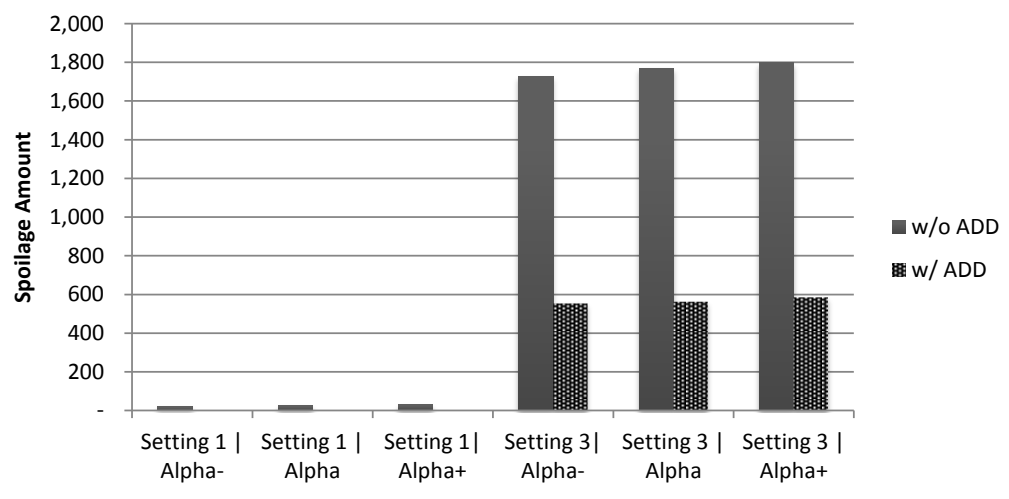

Figure 9: Amount of spoilage taking (or not) into consideration age dependent demand for instances of Settings 1 and 3 when varying $\alpha_{k}$.

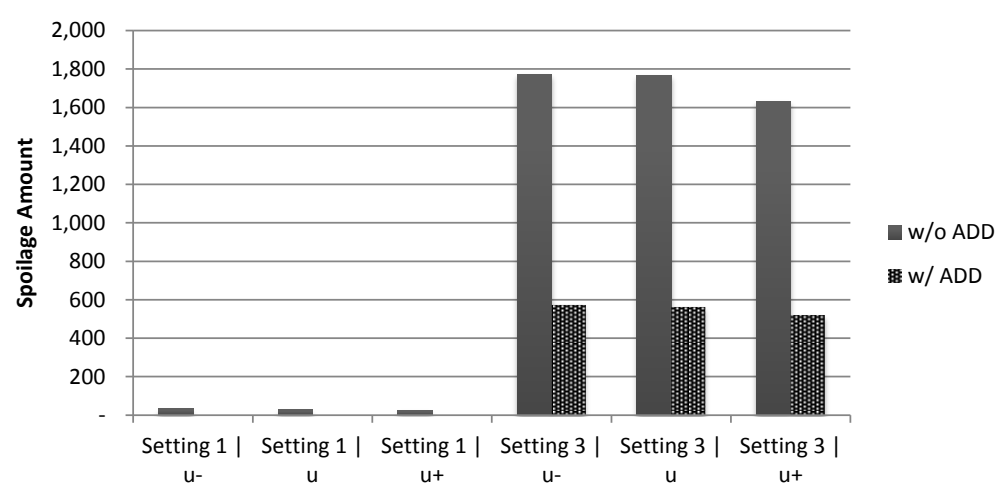

Figure 10: Amount of spoilage taking (or not) into consideration age dependent demand for instances of Settings 1 and 3 when varying $u_{k}$.

severely impacted by the acknowledgement of a decreasing demand throughout the age of the product in the production plans. The potential savings in product spoilage ascend to a complete reduction in the spoiled inventory for Setting 1, which have products with a long shelf-life. For Setting 3, the absolute savings are more significant and the relative savings are close to 70\%. Setting 3, which includes Chicken and Beef products, has a consider- 
able amount of spoilage due to the short shelf-lives, the high price elasticities of demand and the products' high PQR. Overall, the amount of spoilage does not appear to be very sensitive to small variations of the customers' sensitivity and of the products' shelf-lives. In an era of strong environmental awareness both in the civil society and in companies, spoilage is a crucial indicator to be taken into consideration.

\subsubsection{Importance of considering customer's eagerness to pick up the fresher} products $(C F P)$

As previously mentioned, besides incorporating the consumer purchasing behaviour by developing a set of expressions capable of describing demand throughout the shelf-life of products, this study introduces constraints (14) and (15) in the production planning formulation to describe the common customer behaviour of picking the fresher products available. The profit for the solution which includes the mentioned customer behaviour (A) is compared to the solution where the production decision variables are optimised without considering such behaviour (B). Figures 11 and 12 show the percentage objective function loss by not considering the fact that customers pick up the fresher products available.

Results indicate that the profit loss of considering this consumer behaviour is less significant than the loss stemming from not considering the age dependent demand. In fact, while acknowledging the decreasing WTP, there is an implicit assumption on the consumers' preference for fresher products. This will drive production plans towards a leaner strategy and, therefore, this consumer characteristic is implicitly incorporated. Nevertheless, despite the fact that disregarding this consumer behaviour results in a small impact 


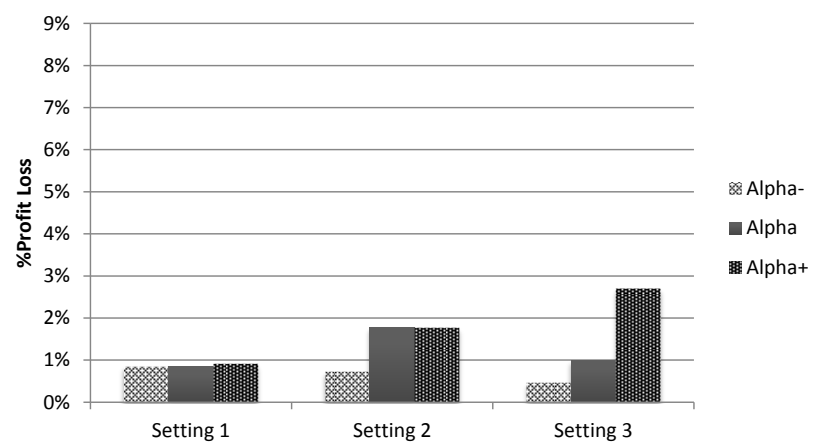

Figure 11: Profit loss due to neglecting consumer purchasing behaviour of picking up the fresher products available for all Settings varying $\alpha_{k}$.

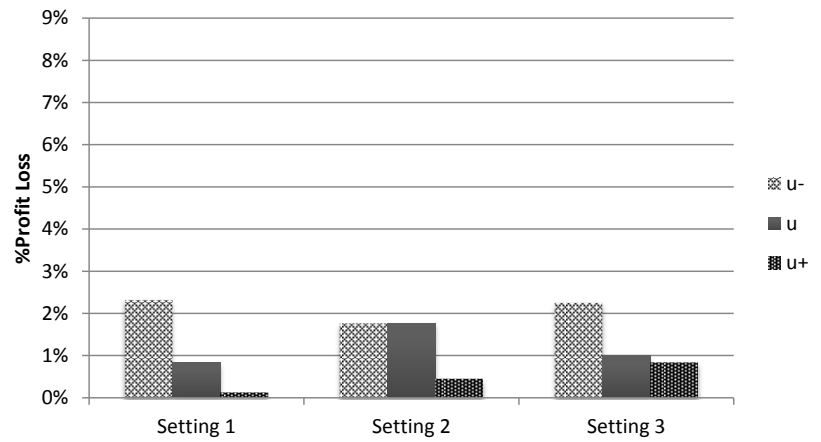

Figure 12: Profit loss due to neglecting consumer purchasing behaviour of picking up the fresher products available for all Settings varying $u_{k}$.

on the profit loss, the solution structure may differ considerably as only the fresher products may be used to meet demand.

\subsubsection{Amount of spoiled products caused by not considering customer's ea-} gerness to pick up the fresher products (CFP)

This analysis of this perspective follows a very similar method to Section 5.2.2. Therefore the stochastic model is also used, but in this case we compare the amount of spoilage for all instances when planning with and without the 
fact that customers are prone to pick up the products with a higher shelf-life. Figures 13 and 14 present the results for all instances when planning with and without this customer characteristic. Notice that once again results for instances of Setting 2 are left out as there was no spoilage incurred.

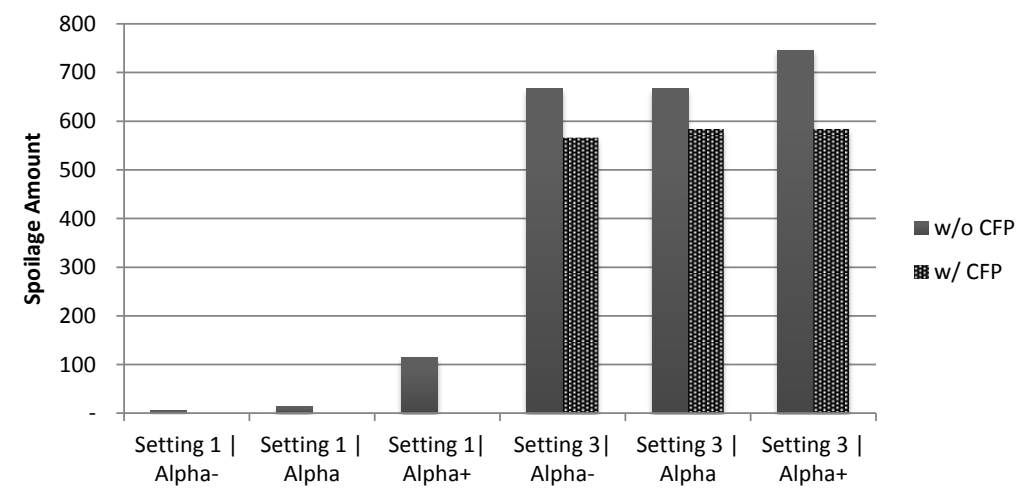

Figure 13: Amount of spoilage taking (or not) into consideration customer's eagerness for fresher products for instances of Settings 1 and 3 when varying $\alpha_{k}$.

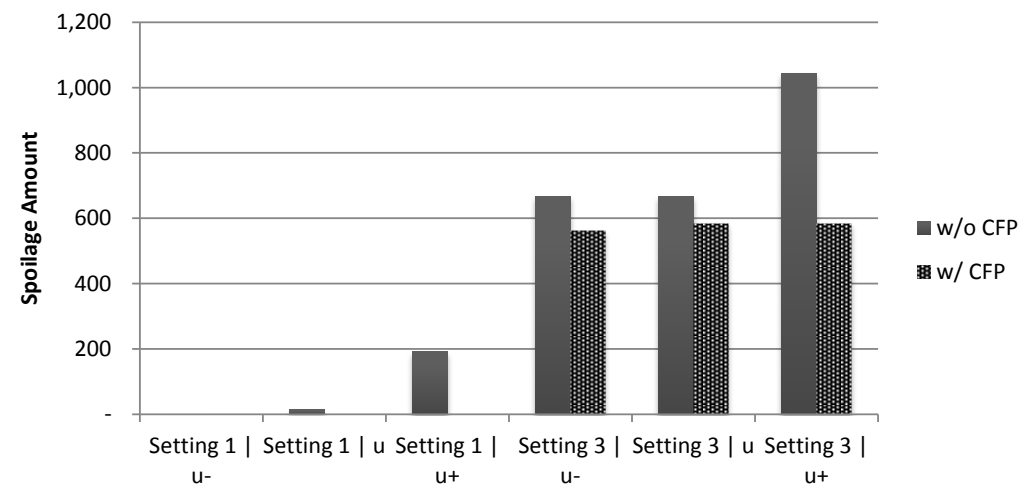

Figure 14: Amount of spoilage taking (or not) into consideration customer's eagerness for fresher products for instances of Settings 1 and 3 when varying $u_{k}$.

The results show that the amount of spoilage can be reduced by incorporating in production planning the fact that customers choose products with 
a highest degree of freshness. As in Section 5.2.2, the potential savings in product spoilage ascend to a complete reduction in the spoiled inventory for Setting 1, but the absolute gains are higher for Setting 3. The increased visibility over this behaviour leads to a better timing of the production lots. Overall, the amount of spoilage does not appear to be very sensitive to small variations of the customers' sensitivity, but products' shelf-lives are relevant to assess the amount of spoilage. With increasing shelf-lives the potential for making wrong choices on the production planning increases.

\subsubsection{Impact of assuming a medium $P Q R$ for all products ( $P Q R$ )}

This analysis aims at understanding the costs resulting from assuming a medium PQR for all products regardless of their nature. The motivation for looking to such perspective comes from the fact that in practical terms it may be rather hard to implement the different equations (8)-(10) for all different products produced by a company. The results obtained in case the production plan assumes a medium PQR for all products are compared against the case where production planning incorporates the correct PQR for all products. For these tests, only Setting 3 is used as the other Settings just have products with medium PQRs. Figure 15 presents the obtained results.

A profit loss below $2.5 \%$ was obtained for all instances, indicating that differentiating between the various PQRs is not as important as differentiating the remaining inputs for the age dependent demand, such as price elasticity of demand, consumer sensitivity or shelf-life. The conclusion of these results is that, in practical applications, food production planners have the plausible option of considering a medium PQR for all products when computing the

age dependent demand functions (Section 3). Moreover, this loss should be 


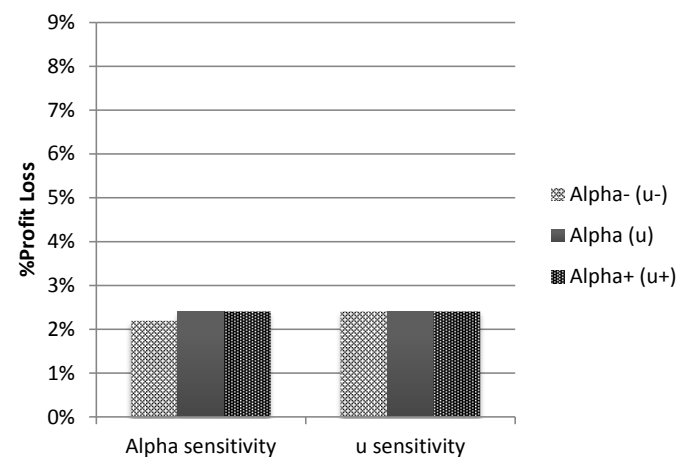

Figure 15: Profit loss due to assuming a medium PQR for all products of Setting 3 varying $\alpha_{k}$ and $u_{k}$.

even smoother in a real-world scenario in which companies produce a mix of products with high and low PQR.

\section{Conclusions and Future Work}

In this study, a set of age dependent demand functions was first developed for products with different PQR based on research analysing consumer purchasing behaviour for perishable food products. A deterministic model was proposed for the production planning of perishable goods that accounts both for decreasing WTP and customers' eagerness to choose the product in a fresher state. This deterministic model is extended to a stochastic model dealing with demand uncertainty, which is an important feature of the fast moving food consumer goods markets. The computational study focuses on a sensitivity analysis in which consumer sensitivity to perishability and product's shelf-life is varied. Results indicate that extending food production planning models to deal with an age dependent demand is of great importance both in terms of profit and product spoilage. Products having high 
consumer sensitivity, a low shelf-life and a high product quality risk are specially important to address. It is expected that in real-world scenarios, these results should hold and even be more evident. In our sensitivity analysis the variations of the parameters were limited to $25 \%$. In real-world cases, one can expect customers with very opposite consumer attitudes towards the ageing of the products (influencing $\alpha_{k}$ ) and storage conditions that vary considerably (influencing $u_{k}$ ).

Future work should focus on different extensions of this problem. Firstly, it is interesting to study the impact of modelling $\beta_{k}$ as a random variable (Nahmias, 1982). Secondly, demand uncertainty provides further motivation to investigate this problem under a risk management perspective. Recent studies have started exploiting the multi-objective problem of considering risk management in production planning (Tometzki \& Engell, 2010). The production planning of perishable goods is, indeed, a very promising field to go further in this direction. Finally, developing a proper solution method that is able to solve larger instances of this problem is of the utmost importance, especially for the stochastic formulation.

\section{Acknowledgements}

The first author appreciate the support of the FCT Grant SFRH/BPD/89861/2012 and the FCT Project PTDC/EGE-GES/104443/2008.

\section{References}

Abad, P. (2001). Optimal price and order size for a reseller under partial backordering. Computers \&3 Operations Research, 28, 53-65. 
Abad, P. L. (2003). Optimal pricing and lot-sizing under conditions of perishability, finite production and partial backordering and lost sale. European Journal of Operational Research, 144, 677-685.

Ahumada, O., \& Villalobos, J. R. (2011). Operational model for planning the harvest and distribution of perishable agricultural products. International Journal of Production Economics, 133, 677-687.

Akkerman, R., Farahani, P., \& Grunow, M. (2010). Quality, safety and sustainability in food distribution: a review of quantitative operations management approaches and challenges. OR Spectrum, 32, 863-904.

Amorim, P., Antunes, C. H., \& Almada-Lobo, B. (2011a). Multi-objective lot-sizing and scheduling dealing with perishability issues. Industrial $\&$ Engineering Chemistry Research, 50, 3371-3381.

Amorim, P., Günther, H.-O., \& Almada-Lobo, B. (2012). Multi-objective integrated production and distribution planning of perishable products. International Journal of Production Economics, 138, 89 - 101.

Amorim, P., Meyr, H., Almeder, C., \& Almada-Lobo, B. (2011b). Managing perishability in production-distribution planning: a discussion and review. Flexible Services and Manufacturing Journal, (pp. 1-25). DOI:10.1007/s10696-011-9122-3.

Andreyeva, T., Long, M. W., \& Brownell, K. D. (2010). The impact of food prices on consumption: a systematic review of research on the price elasticity of demand for food. American Journal of Public Health, 100, $216-22$. 
Bilgen, B., \& Günther, H.-O. (2009). Integrated production and distribution planning in the fast moving consumer goods industry: a block planning application. OR Spectrum, 32, 927-955.

Birge, J. R., \& Louveaux, F. (2011). Springer series in operations research. In Introduction to Stochastic Programming. Springer: New York.

Broekmeulen, R. A., \& van Donselaar, K. H. (2009). A heuristic to manage perishable inventory with batch ordering, positive lead-times, and timevarying demand. Computers $\&$ Operations Research, 36, 3013 - 3018.

Buschkühl, L., Sahling, F., Helber, S., \& Tempelmeier, H. (2008). Dynamic capacitated lot-sizing problems: a classification and review of solution approaches. OR Spectrum, 32, 231-261.

Chang, H., Teng, J., Ouyang, L., \& Dye, C. (2006). Retailer's optimal pricing and lot-sizing policies for deteriorating items with partial backlogging. European Journal of Operational Research, 168, 51-64.

Chen, J., \& Chen, L. (2005). Pricing and production lot-size/scheduling with finite capacity for a deteriorating item over a finite horizon. Computers $\&$ Operations Research, 32, 2801-2819.

Costa, A. M., Santos, L. M. R., Alem, D. J., \& Santos, R. H. S. (2013). Sustainable vegetable crop supply problem with perishable stocks. Annals of Operations Research, (pp. 1-19).

Drexl, A., \& Kimms, A. (1997). Lot sizing and scheduling Survey and extensions. European Journal of Operational Research, 99, 221-235. 
Dyllick, T. (1989). Ecological marketing strategy for Toni yogurts in Switzerland. Journal of Business Ethics, 8, 657-662.

Fleischmann, B., \& Meyr, H. (1997). The general lotsizing and scheduling problem. OR Spektrum, 19, 11-21.

Günther, H.-O., Grunow, M., \& Neuhaus, U. (2006). Realizing block planning concepts in make-and-pack production using MILP modelling and SAP APO . International Journal of Production Research, 44, 3711-3726.

Haase, K., \& Kimms, A. (2000). Lot sizing and scheduling with sequencedependent setup costs and times and efficient rescheduling opportunities. International Journal of Production Economics, 66, 159-169.

Jans, R., \& Degraeve, Z. (2008). Modeling industrial lot sizing problems: a review. International Journal of Production Research, 46, 1619-1643.

Karaesmen, I., Scheller-Wolf, A., \& Deniz, B. (2011). Managing perishable and aging inventories: review and future research directions. In K. Kempf, P. Keskinocak, \& R. Uzsoy (Eds.), Handbook of Production Planning Kluwer International Series in Operations Research and Management Science. Kluwer Academic Publishers, Dordrecht.

Karimi, B., Fatemi Ghomi, S., \& Wilson, J. (2003). The capacitated lot sizing problem: a review of models and algorithms. Omega, 31, 365-378.

Krarup, J., \& Bilde, O. (1977). Plant location, set covering and economic lot sizes: an $O(m n)$ algorithm for structured problems. L. Collatz et al. eds, Birkhauser Verlag: Basel, Switzerland. 
Lütke Entrup, M. (2005). Shelf life integration in sausage production. In Advanced Planning in Fresh Food Industries Contributions to Management Science (pp. 179-195). Physica-Verlag HD.

Lütke Entrup, M., Günther, H.-O., Van Beek, P., Grunow, M., \& Seiler, T. (2005). Mixed-Integer Linear Programming approaches to shelf-lifeintegrated planning and scheduling in yoghurt production. International Journal of Production Research, 43, 5071-5100.

Marinelli, F., Nenni, M. E., \& Sforza, A. (2007). Capacitated lot sizing and scheduling with parallel machines and shared buffers: A case study in a packaging company. Annals of Operations Research, 150, 177-192.

Nahmias, S. (1982). Perishable inventory theory: a review. Operational Research, 30, 680-708.

Otto, A., Schoppengerd, F. J., \& Shariatmadari, R. (2009). Success in the consumer products market: Understanding direct store delivery. In R. Shariatmadari, F. J. Schoppengerd, \& A. Otto (Eds.), Direct Store Delivery (pp. 1-29). Springer Berlin Heidelberg.

Pahl, J., \& Voß, S. (2010). Discrete Lot-Sizing and Scheduling Including Deterioration and Perishability Constraints. In W. Dangelmaier, A. Blecken, R. Delius, \& S. Klöpfer (Eds.), Advanced Manufacturing and Sustainable Logistics (pp. 345-357). Springer Berlin Heidelberg volume 46 of Lecture Notes in Business Information Processing.

Pahl, J., Voß, S., \& Woodruff, D. L. (2011). Discrete Lot-Sizing and Schedul- 
ing with Sequence-Dependent Setup Times and Costs including Deterioration and Perishability Constraints. In IEEE, HICSS-44.

Rong, A., Akkerman, R., \& Grunow, M. (2011). An optimization approach for managing fresh food quality throughout the supply chain. International Journal of Production Economics, 131, 421-429.

Sen, S., \& Block, L. G. (2009). "Why My Mother Never Threw Anything Out": The Effect of Product Freshness on Consumption. Journal of Consumer Research, 36, 47-55.

Sodhi, M. (2005). Managing demand risk in tactical supply chain planning for a global consumer electronics company. Production and Operations Management, 14, $69-79$.

Tometzki, T., \& Engell, S. (2010). Risk Management in Production Planning under Uncertainty by Multi-Objective Hybrid Evolutionary Algorithms. In Computer Aided Chemical Engineering (pp. 151-156). Elsevier volume 28.

Tsiros, M., \& Heilman, C. M. (2005). The Effect of Expiration Dates and Perceived Risk on Purchasing Behavior in Grocery Store Perishable Categories. Journal of Marketing, 69, 114-129.

Van Woensel, T., Van Donselaar, K., Broekmeulen, R., \& Fransoo, J. (2007). Consumer responses to shelf out-of-stocks of perishable products. International Journal of Physical Distribution 83 Logistics Management, 37, 704-718.

Zhu, X., \& Wilhelm, W. (2006). Scheduling and lot sizing with sequencedependent setup: A literature review. IIE Transactions, 38, 987-1007. 


\section{University Library}

\section{- M M I E E R VA A gateway to Melbourne's research publications}

Minerva Access is the Institutional Repository of The University of Melbourne

Author/s:

Amorim, P;Costa, AM;Almada-Lobo, B

Title:

Influence of consumer purchasing behaviour on the production planning of perishable food

Date:

2014-07-01

Citation:

Amorim, P., Costa, A. M. \& Almada-Lobo, B. (2014). Influence of consumer purchasing behaviour on the production planning of perishable food. OR SPECTRUM, 36 (3), pp.669-692. https://doi.org/10.1007/s00291-013-0324-9.

Persistent Link:

http://hdl.handle.net/11343/282964 